\title{
Immunology of SARS-CoV-2 infection in children
}

\author{
Janet Chou', Paul G. Thomas $\mathbb{( i}^{2}$ and Adrienne G. Randolph $\oplus^{3 凶}$
}

\begin{abstract}
Children and adolescents exhibit a broad range of clinical outcomes from SARS-CoV-2 infection, with the majority having minimal to mild symptoms. Additionally, some succumb to a severe hyperinflammatory post-infectious complication called multisystem inflammatory syndrome in children (MIS-C), predominantly affecting previously healthy individuals. Studies characterizing the immunological differences associated with these clinical outcomes have identified pathways important for host immunity to SARS-CoV-2 and innate modulators of disease severity. In this Review, we delineate the immunological mechanisms underlying the spectrum of pediatric immune response to SARS-CoV-2 infection in comparison with that of adults.
\end{abstract}

A $\mathrm{t}$ the start of the severe acute respiratory syndrome coronavirus 2 (SARS-CoV-2) pandemic, most children were initially spared, with very few developing moderate to severe coronavirus disease 2019 (COVID-19) ${ }^{1-7}$. As the virus spread globally, the majority of children $<18$ years of age had asymptomatic infection or mild COVID-19, and hospitalization was are $^{8-12}$. Those children who did develop severe COVID-19 generally had risk factors, including underlying respiratory, neurological, or immune disorders ${ }^{13}$. This pattern contrasts with other respiratory viruses, including respiratory syncytial virus (RSV), influenza virus, and parainfluenza virus, known to cause severe disease in young children $^{14,15}$. In April 2020, a post-infectious syndrome known as multisystem inflammatory syndrome in children (MIS-C) emerged in individuals $<21$ years of age, with the majority of patients requiring intensive care for life-threatening complications. Investigating age-associated determinants of the spectrum of clinical outcomes in children and adults related to SARS-CoV-2 infection is paramount for understanding host susceptibility and outcome and could help optimize disease prevention and treatment.

This Review synthesizes important principles of developmental immunology with evidence linking immunobiology and clinical outcomes of SARS-CoV-2 infections in children and adults. Because severe COVID-19 and MIS-C are uncommon in children, sample sizes for published studies are limited. To address this limitation, we highlight findings that are common as well as disparate across published studies, with the goal of identifying fundamental distinctions between the pediatric and adult response to SARS-CoV-2 infections. Owing to the spectrum of disease associated with SARS-CoV-2 infections, we compare the immunological response of children and adults within distinct clinical phenotypes. These phenotypes include asymptomatic SARS-CoV-2 infections, mild COVID-19 (characterized by the presence of upper respiratory symptoms not requiring supportive care), and symptomatic COVID-19 prompting medical attention. Within each section, we discuss hypotheses that have been investigated to explain why children are more protected from moderate to severe COVID-19 than adults.

\section{Asymptomatic SARS-CoV-2 infection to mild COVID-19}

Overall, studies thus far have shown that responses to asymptomatic or mild COVID-19 are similar between adults and children. Three studies found that the viral loads of children with asymptomatic SARS-CoV-2 infection or mild disease are comparable ${ }^{16}$ to, or slightly lower than, viral loads in adults with similarly mild SARS-CoV-2 infections ${ }^{17,18}$. Jia et al. showed that the cytokine profiles of children with mild COVID-19 resemble profiles from healthy children, reflecting a low level of inflammation ${ }^{19}$. Similarly, Cohen et al. found no differences in the percentage of interferon- $\gamma$ (IFN- $\gamma$ )-producing $\mathrm{CD}^{+}$or $\mathrm{CD}^{+} \mathrm{T}$ cells with $\mathrm{T}$ cell receptors (TCR) specific for structural, accessory, and non-structural proteins in nine asymptomatic children compared with that in nine adults, with comparable capacities for generating multiple cytokines ${ }^{20}$. This similarity occurred despite a reduced level of cross-reactive antibodies to $\beta$-coronaviruses other than SARS-CoV-2 in children compared with adults with similarly mild infections, suggesting that differences in prior infections do not substantially affect the T cell response in individuals with mild SARS-CoV-2 infections ${ }^{20}$.

Parallel to a robust cellular response to SARS-CoV-2, children are capable of mounting a robust serological response to mild SARS-CoV-2 infections. A study showed that children, adolescents, and adults with mild COVID-19 had similar levels of anti-spike IgG with comparable functionality, including the capacity for activating complement and phagocytic cells ${ }^{21}$. Two studies showed even higher neutralizing activity in anti-SARS-CoV-2 antibodies from children $<10$ years of age than in adolescents and young adults ${ }^{22,23}$. The effect of disease severity on the antibody response is indicated by lower levels of convalescent antibodies against the spike and nucleocapsid proteins in children with asymptomatic or mild infections than in those with moderate COVID-19 requiring hospitalization $^{24,25}$. Collectively, these studies do not show major differences in the viral load or immune response to mild COVID-19 between children and adults.

\section{Moderate to severe COVID-19}

In contrast with the comparable pediatric and adult immune responses during mild COVID-19, notable differences occur in patients hospitalized with COVID-19. Even though the number of pediatric individuals with COVID-19 increased after the spread of the Delta variant ${ }^{26}$, compared with hospitalized adults, most children and adolescents hospitalized for COVID-19 required shorter hospitalizations and less respiratory support, and mortality was less com$\operatorname{mon}^{27,28}$. In the USA, individuals $>50$ years of age account for at least $90 \%$ of all deaths ${ }^{29}$. Differences in immune responses could be either predictive or reflective of COVID-19 disease severity. Additionally, comorbid conditions differ between adults and children, as do alcohol and tobacco use and obesity, which can all affect immune outcomes. In this section, we discuss the differences in the immune systems of children and adults that have been investigated as potential drivers of age-associated clinical outcomes from COVID-19 (Fig. 1). 


\section{Fewer comorbidities}

Obesity

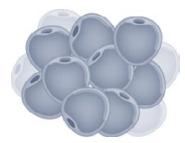

Chronic liver disease

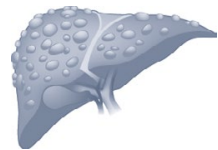

Innate

immunity

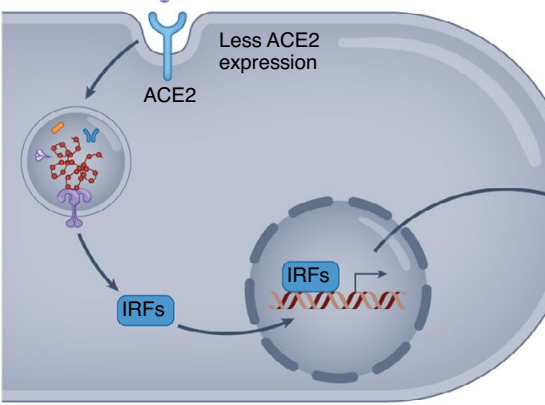

T cell immunity
Diabetes

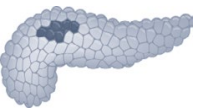

Chronic lung disease

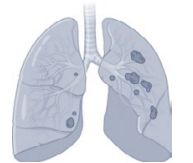

Neurological disease

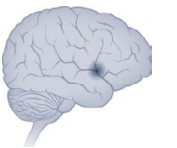

Chronic kidney disease

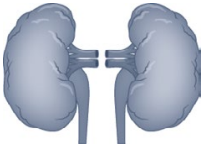

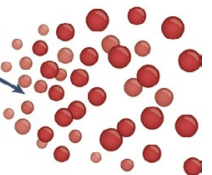

Robust interferon response

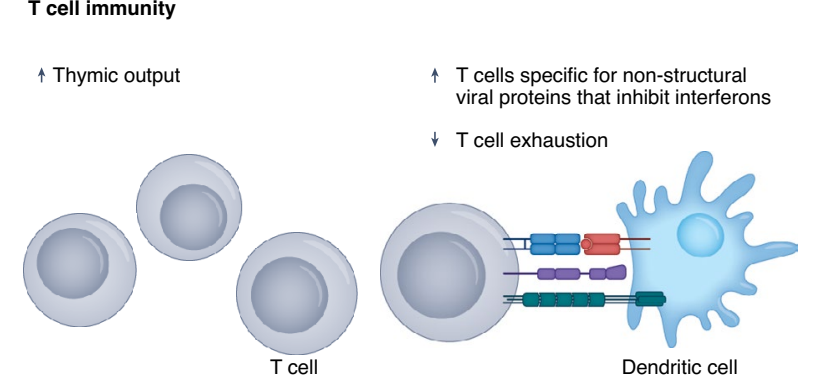

Fig. 1 | Factors that reduce the severity of SARS-CoV-2 infection in children. Children tend to have fewer comorbidities than adults. Protective innate immune factors include reduced expression of ACE2 and a more robust IFN response. Compared with adults, children also have more T cells owing to greater thymic output, more $T$ cells that recognize non-structural viral proteins, and less $T$ cell exhaustion.

\section{Potential contributors to differences in adult versus pediatric COVID-19}

In this section, we review studies pointing to developmental differences in innate and adaptive immunity (Fig. 2). Studies have focused particularly on differences in IFN signaling and $\mathrm{T}$ cell function as contributors to the increased susceptibility to severe COVID-19 in adults. In support of this idea, the prevalence of severe COVID-19 was higher in immunodeficient cohorts enriched for disorders of $\mathrm{T}$ cell development and/or function ${ }^{30-32}$, compared with that of the general pediatric population or cohorts with a broader diversity of primary or secondary immunodeficiencies, such as antibody deficiencies, autoimmune disorders, or those being treated with immunosuppressive medications $s^{6,33,34}$. The increased frequency of severe COVID-19 in individuals with reduced $\mathrm{T}$ cell numbers parallels the increased mortality from COVID-19 in older people, another population with reduced $\mathrm{T}$ cell number and function ${ }^{35,36}$. Notably, comorbidities associated with severe COVID-19 (ref. ${ }^{37}$ ), including cardiovascular disease, diabetes, malignancies, renal failure, and neurodegenerative disease, occur more frequently in adults than in children. Some chronic diseases, such as cerebrovascular disease, obesity, and diabetes, can result in a dysfunctional immune response to infection through epigenetic and metabolic reprogramming of immune cells ${ }^{38-40}$. Other comorbidities, such as chronic lung disease, can result in anatomical susceptibility to more-severe COVID-19. When present in children and young adults, these comorbidities also increase the risk of severe COVID-19 (refs. ${ }^{6,9,10,13,41}$ ).

SARS-CoV-2 entry factors. Upon encountering respiratory mucosa, the receptor-binding domain (RBD) of the SARS-CoV-2 spike protein binds to the widely expressed angiotensin-converting enzyme 2 (ACE2) receptor ${ }^{42-44}$. The subsequent fusion of viral and cellular membranes is facilitated by cleavage of the viral spike protein by host proteases, such as transmembrane serine protease 2 (TMPRSS2) and cathepsin L, thereby enabling cellular entry $y^{45,46}$. TMPRSS2 expression in the airway epithelium increases with age ${ }^{47-50}$. A meta-analysis of 31 single-cell RNA-sequencing studies from 228 individuals without a history of lung disease also showed a positive correlation between age and the expression of ACE2 in alveolar cells $^{51}$. However, distinctions in the expression of viral entry factors between children and adults are less clear, as only some studies support the hypothesis that fewer ACE2 receptors in children can account for reduced viral entry into the lungs $\mathrm{s}^{4,52,53}$.

In adults with COVID-19, higher viral loads measured through nasopharyngeal or oropharyngeal swabs have been associated with more-severe disease in some, but not all, studies, particularly as asymptomatic individuals might have viral loads comparable to those found in hospitalized individuals ${ }^{1,54-57}$. Although studies have detected higher viral loads in symptomatic children than in asymptomatic children, viral loads have not been predictive of disease severity in children ${ }^{58,59}$. Furthermore, children and adults with similar disease severity have comparable viral loads ${ }^{16,17}$. The variability in these studies might arise from differences in collection techniques or the inability to sample the lower respiratory tract in non-intubated individuals. Nonetheless, studies thus far have not conclusively shown that differences in viral load account for improved clinical outcomes that occur in the majority of children with COVID-19.

Interferon signaling. Although the ACE2 receptor is critical for SARS-CoV-2 cellular entry, other viral components activate widely expressed pattern-recognition receptors to initiate the innate immune response $e^{60}$. SARS-CoV-2 envelope protein and spike protein are sensed by Toll-like receptor 2 (TLR2) and TLR4, respectively ${ }^{60,61}$. Single-stranded viral genomic RNA activates TLR7/8, while the virus's double-stranded RNA intermediates bind TLR3, and cytosolic RNA is sensed by the RIG-I-like receptors ${ }^{62}$. Activation of TLRs and RIG-I induces a robust IFN response. As a counter-regulatory mechanism, SARS-CoV-2 proteins, such as ORF9b, non-structural proteins 1 and 13, and the nucleocapsid protein, can inhibit the host IFN response ${ }^{62,63}$. Differences in IFN signaling have been investigated in children and adults with COVID-19. Adults with severe COVID-19 have lower circulating levels of type I IFNs, which has been attributed to neutralizing antibodies to type I IFNs ${ }^{64-66}$, as well as to reduced type I IFN secretion from plasmacytoid dendritic $\operatorname{cells}^{67,68}$. Pierce et al. compared the immune response in nasal secretions from 12 children and 27 adults hospitalized for moderate to severe COVID-19; children had much milder disease than the adults, requiring no respiratory support and resulting in no mortalit $^{52}$. Compared with adults, the children in this study had increased expression of genes downstream of type I and II IFNs and the NLR family pyrin domain-containing 3 (NLRP3) inflammasome, 


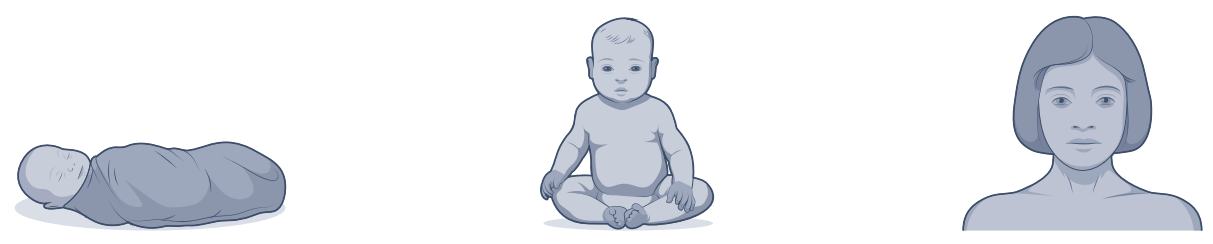

\begin{tabular}{|l|l|}
\hline Innate & $\begin{array}{l}\text { IFN, IL-12, IL-10 } \\
\text { Innate cell function }\end{array}$ \\
\hline Humoral & $\begin{array}{l}\text { Development of germinal } \\
\text { centers, T cell-independent } \\
\text { antibody response, somatic } \\
\text { hypermutation }\end{array}$ \\
\hline Cellular & $\begin{array}{l}\text { TCR repertoire diversity } \\
\text { T cell function }\end{array}$ \\
& Frequency of regulatory T cells \\
\hline
\end{tabular}

Fig. 2 | Developmental differences in pediatric and adult immune response to viral infection. Innate cell function reaches adult levels in early childhood. B cell and $T$ cell function continues to develop throughout childhood. By contrast, regulatory $T$ cell function wanes with aging.

but both groups had similar viral loads and anti-SARS-CoV-2 IgG and IgA titers in nasal fluid ${ }^{52}$. In correlating the increased IFN and inflammasome signaling in the nasal mucosa with better clinical outcomes, this study further supports the hypothesis that children are protected from severe disease owing to more robust innate immune responses that enable them to overcome SARS-CoV-2. One caveat is that upper respiratory samples might not represent the cellular composition and gene expression of cells and fluid from lower airways or peripheral blood ${ }^{69,70}$.

The heightened IFN response in children does not arise exclusively from intrinsic age-associated differences in this pathway. IFN responses are reduced from the time of birth until approximately 2 years of age. Then, between 2 and 5 years of age, the serum cytokine profiles shift toward that of an adult, with increased expression of type I IFNs, reduced IL-6 and IL-23, and increased IL-12 and IL-10 (refs. ${ }^{71,72}$ ). A reduction in the IFN response occurs again in adults $>65$ years of age, in part due to age-associated reductions in interferon-regulatory factor 8 (IRF8), which is a transcription factor downstream of RIG-I signaling, and proteolysis of tumor receptor-associated factor 3 (TRAF3), which is a signaling protein utilized by multiple TLRs ${ }^{73}$. Additionally, as individuals age, type I IFN receptors in $\mathrm{CD}^{+} \mathrm{T}$ cells are more frequently in complex with Src homology region 2 domain-containing phosphatase 1 (SHP-1), an inhibitory protein tyrosine phosphatase that restrains type I IFN signaling ${ }^{74,75}$. Exogenous factors that reduce the IFN response also accumulate with age. These factors include chronic viral infections, such as human cytomegalovirus, known to cause exhaustion of plasmacytoid dendritic cells and reduced IFN secretion, as well as anti-IFN autoantibodies $^{64,76-78}$. Although the effects of reduced IFN signaling are most pronounced in individuals $>65$ years of age, these factors progressively impair the IFN response and might contribute to the increased frequency of severe COVID-19 in middle-aged and older adults.

T cell homeostasis and activation. $T$ cell lymphopenia in the blood and airway fluid is a marker of COVID-19 severity in children and adults ${ }^{52,70,79}$. In a study comparing the immune response of 65 children and young adults under 24 years of age with that of 60 adults, all of whom were hospitalized for COVID-19, children had higher absolute lymphocyte counts ${ }^{27}$. However, compared with children and infants, adults generated increased percentages of spike-specific IFN- $\gamma^{+} \mathrm{CD} 4^{+} \mathrm{T}$ cells ${ }^{27}$. In another study analyzing $\mathrm{T}$ cell responses (with 34 children and 36 adults), the overall magnitude of SARS-CoV-2-specific $\mathrm{CD}^{+}$and $\mathrm{CD}^{+} \mathrm{T}$ cell responses was lower in the blood of children than in that of adults ${ }^{20}$. The TCR specificities of CD4 ${ }^{+} \mathrm{T}$ cells varied widely between children and adults, with adult $T$ cells being specific mainly for structural proteins. By contrast, $\mathrm{CD} 4^{+} \mathrm{T}$ cells from children consisted of a substantial proportion that were specific for open reading frame $1 \mathrm{ab}$ (Orf1ab), which encodes 16 non-structural proteins ${ }^{20}$. As some of these non-structural proteins are known to block the host IFN response $^{63}$, these studies raise the possibility that differences in $\mathrm{T}$ cell specificity might contribute to the age-associated decrease in the IFN response to SARS-CoV-2 infection.

Given the dynamic changes affecting $\mathrm{T}$ cell development throughout life, multiple age-related differences in $\mathrm{T}$ cell number and function probably contribute to the increased frequency of severe COVID-19 in adults relative to that in children. Thymic output is maximal at 1 year of age, and decreases gradually thereafter, with negligible output occurring by $\sim 85$ years of age ${ }^{80}$. The TCR repertoire is another factor that evolves throughout a lifetime. Pathogen-specific memory responses expand as infants and young children encounter new infections, including the formation of tissue-resident memory $\mathrm{T}$ cells ${ }^{81}$. Although some studies have identified T cell cross-reactivity to epitopes of SARS-CoV-2 and those of other human coronaviruses, a definitive correlation between clinical outcomes and $\mathrm{T}$ cell epitope cross-reactivity has not been made ${ }^{82}$. Aging is also associated with shifts in the transcriptional profiles of $\mathrm{T}$ cells. Aging mice housed in specific-pathogen-free environments accumulate cytotoxic and effector $\mathrm{CD}^{+}{ }^{+} \mathrm{T}$ cells, with increased expression of genes associated with chronic inflammation (such as S100 protein family members), the cytotoxic $\mathrm{T}$ cell response to viral infections (such as granzymes and the transcription factor eomesodermin), and $\mathrm{T}$ cell exhaustion ${ }^{83}$. The similarities between $\mathrm{T}$ cell dysfunction in aging adults and in those with severe COVID-19 suggest that the gradual onset of $\mathrm{T}$ cell dysfunction in adulthood might set the stage for further dysfunctional $\mathrm{T}$ cell responses that are associated with severe COVID-19. Although some studies have delved deeply into mechanisms of $\mathrm{T}$ cell dysfunction in older people ${ }^{84,85}$, additional investigations are needed to delineate the threshold of $\mathrm{T}$ cell dysfunction associated with susceptibility to severe COVID-19.

Serological responses. SARS-CoV-2 drives the expansion of plasmablasts and plasma cells during both mild and severe COVID-19, even more so than influenza vaccination and infection ${ }^{86-89}$. Children 
and adults hospitalized with COVID-19 have comparable levels of plasmablasts, regardless of need for ventilatory support ${ }^{90}$. Increased plasmablast frequencies correlate with percentages of proliferating $\mathrm{CD}^{+} \mathrm{T}$ cells, rather than with levels of anti-spike antibodies ${ }^{90,91}$. Adults with acute severe COVID-19 exhibited increased levels of neutralizing antibodies compared with titers from convalescent adults or pediatric individuals with MIS-C, the latter two of which were comparable in one study ${ }^{70}$.

The differences in the serological response to COVID-19 in children and adults are concordant with known age-associated differences in B cell development (Fig. 1). The response to T cellindependent antigens takes $\sim 2$ years to mature, as infants produce more IgG1 than IgG2, the latter of which preferentially recognizes polysaccharide antigens ${ }^{92,93}$. Environmental factors also shape B cell development. Clonal expansion and somatic hypermutation begin during infancy and increase with environmental and infectious exposures $^{93-95}$. The rate of somatic hypermutation can be increased, even during infancy, through exposure to infectious pathogens ${ }^{94}$. However, also important to note is that the severity of COVID-19 in adults also affects the serological response ${ }^{8-12}$. Indeed, Bartsch et al. showed that adults with severe COVID-19 have increased titers of anti-SARS-CoV-2 IgG and IgA with more functionality in activating complement and phagocytic cells, compared with either adults or children with mild SARS-CoV-2 infections ${ }^{21}$.

Antibody repertoires also evolve with age and infectious exposures. With respect to other common coronaviruses, including 229E, NL63, OC43, and KHU1, a study of 231 children and 1,168 adults found that children had increased levels of antibodies against the spike, nucleocapsid, and matrix structural proteins, whereas a non-structural protein of unknown function was the most common target of antibodies in adults ${ }^{96}$. Age-associated differences in epitope reactivity have also been identified in antibodies against other respiratory viruses, including RSV and influenza virus, suggesting that serological immunity is achieved through targeting different viral epitopes in children than in adults ${ }^{97-100}$. These differences have been attributed, at least in part, to original antigenic sin or immunological imprinting, in which exposures to specific pathogen epitopes skew subsequent antibody repertoires as a result of the reliance of adaptive immunity on existing memory cells ${ }^{100}$. Antigenic sin has been shown to contribute to differences in age-associated mortality with influenza virus subtypes ${ }^{101,102}$. For the response to COVID-19, no definitive associations have been made between prior coronavirus infections and the serological response to SARS-CoV-2. A study of 37 adults with COVID-19 found that serological responses to the spike protein of other betacoronaviruses were inversely proportional to IgM and IgG antibodies against the spike and nucleocapsid proteins of SARS-CoV-2 (ref. ${ }^{103}$ ). Similarly, a study of 232 people with COVID19 identified a negative correlation between antibody responses to SARS-CoV-2 and other human coronaviruses ${ }^{104}$. By contrast, a study of individuals hospitalized for COVID-19 (10 young adults 27-39 years of age and 20 adults 69-82 years of age, of whom 9 required ventilation) found positive correlations between antibodies to the spike protein of other human betacoronaviruses and SARS-CoV-2; serological memory to earlier coronaviruses did not correlate with clinical outcomes ${ }^{105}$. Another study of 65 children and 60 adults did not find significant correlations between serological memory to other human coronaviruses and either age or outcome ${ }^{27}$.

The heterogeneous outcomes of these studies indicate the complexity of investigating immunological imprinting in diverse human populations with a broad range of environmental and infectious exposures. Future studies with animal models of serial viral infections might provide more-definitive conclusions.

\section{MIS-C}

In April 2020, in the wake of viral surges occurring in Italy and the $\mathrm{UK}^{106-109}$, a number of children presented with mucocutaneous features resembling Kawasaki disease or toxic shock syndrome. Soon after, descriptive reports by Feldstein et al. and Dufort et al., totaling almost 300 cases in the USA, confirmed that the timing was $\sim 4$ weeks after the peak of SARS-CoV-2 activity in these regions ${ }^{110,111}$. This disease entity is now referred to as pediatric inflammatory multisystem syndrome temporally associated with SARS-CoV-2 (PIMS-TS) $)^{108}$ or MIS-C ${ }^{112}$.

Symptoms and diagnosis. The diagnostic criteria from the US Centers for Disease Control for MIS-C include at least 24 hours of fever and inflammation severe enough to affect at least two organ systems and require hospitalization in an individual $<21$ years of age who has no alternative diagnosis explaining the illness ${ }^{112}$. These symptoms must occur during a current or recent SARS-CoV-2 infection or within 4 weeks of exposure to a confirmed COVID-19 case $^{112}$. At least $75 \%$ of children in published cohorts have positive SARS-CoV-2 antibody testing, but fewer (up to 52\%) have positive respiratory testing, suggesting the post-infectious timing of MIS-C $\mathrm{C}^{109-111,113}$. Milder symptoms of MIS-C include fever, rash, and gastrointestinal symptoms, but it can progress to life-threatening cytopenias, coagulopathy, myocardial dysfunction, coronary aneurysms, and shock ${ }^{1-5}$. The majority of children diagnosed with MIS-C were previously healthy and most were critically ill, mostly from shock and/or left ventricular dysfunction, with less-severe or no respiratory involvement ${ }^{110}$. Approximately $40 \%$ of the children met criteria for Kawasaki disease, a vasculitic syndrome associated with coronary artery aneurysms ${ }^{110}$. In a study of $>1,000$ children with MIS-C or severe COVID-19, those with MIS-C were more likely to have cardiovascular and/or mucocutaneous involvement, with higher CRP levels and a higher ratio of neutrophils to lymphocytes $^{113}$. MIS-C predominantly affects those between 6 and 12 years of age, rather than younger or older individuals ${ }^{113,114}$. MIS-C has been reported in Europe, North and South America, the Middle East, South Africa, and South Asia ${ }^{115}$. Relatively fewer cases have been reported from East Asia, which has a high frequency of Kawasaki disease, and it is unclear whether this is due to differences in the genetic background of local populations or environmental conditions $s^{115,116}$. Compared with non-Hispanic white children in the USA, MIS-C occurs more frequently than COVID-19 in non-Hispanic Black children ${ }^{113,114}$.

Immune cell activation in MIS-C. Cellular activation affecting multiple hematopoietic lineages is a consistent feature of MIS-C. High levels of inflammatory cytokines, including IFN- $\alpha$, IFN- $\gamma$, IL-1 $\beta$, IL-6, IL-8, IL-10, and IL-17, have been identified in all studies of MIS-C $\mathrm{C}^{117-123}$, but no single archetypal cytokine signature of MIS-C has emerged. Evidence of myeloid cell activation in children with MIS-C includes increased expression of CD64 on monocytes and neutrophils, as well as of the alarmin-related S100A genes, both of which are also elevated in adults with severe COVID-19 (refs. ${ }^{91,117}$ ). Natural killer cells from children with MIS-C have also been shown to be highly active, with higher expression of perforin and granzymes than in those from healthy adults ${ }^{91}$. Single-cell RNA-seq of peripheral blood mononuclear cells from eight children with MIS-C, compared with four children with COVID-19 pneumonia and nine healthy adults, showed that those with MIS-C and COVID-19 had similarly increased expression of genes downstream of inflammatory cytokines (type I/II IFNs, IL-1, IL-6, and IL-17) and NF- $\kappa$ B activation, even though all children with MIS-C had been treated with immunomodulatory therapies prior to study enrollment ${ }^{123}$. The inflammatory signature in the MIS-C cohort was enriched for monocytes and dendritic cells, highlighting the contribution of these cells to the systemic inflammation that is characteristic of MIS-C $\mathrm{C}^{123}$.

Commensurate with multilineage immune activation, children with MIS-C have tissue inflammation, including increased 


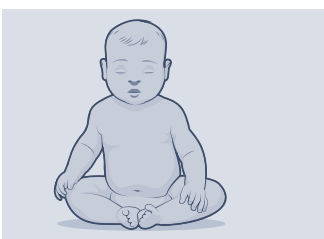

Mild COVID-19

Moderate to severe COVID-19

+ SARS-CoV-2-specific T cells + Anti-SARS-CoV-2 IgM and IgG

\pm Inflammatory cytokines
+ Type I/II IFN

+ Other inflammatory cytokines

+ SARS-CoV-2-specific T cells

++ Follicular helper T cells

++ Anti-SARS-CoV-2 IgG

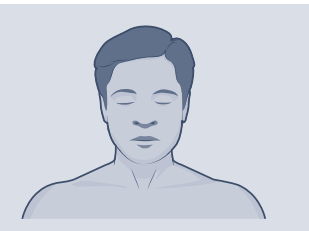

+ SARS-CoV-2-specific T cells

+ Anti-SARS-CoV-2 IgM and IgG

\pm Inflammatory cytokines
- Type I IFN signaling

+++ Other inflammatory cytokines

+++ T cell lymphopenia

+++ SARS-CoV-2-specific T cells

\pm Follicular helper $T$ cells

+++ Anti-SARS-CoV-2 lgG

Fig. 3 | Comparison of the immune response to SARS-CoV-2 in children and adults. Age-related differences in the immune response to SARS-CoV-2

infection are most apparent in moderate to severe COVID-19 and MIS-C.

gastrointestinal permeability and schistocytes; elevated circulating levels of complement $\mathrm{C} 5 \mathrm{~b} 9$, which is indicative of microangiopathy; and release of troponin and natriuretic peptides, indicative of cardiac inflammation ${ }^{109-111,113,118,121,122,124}$. Children with MIS-C might also have mucocutaneous inflammation and coronary artery aneurysms, features characteristic of Kawasaki disease. However, studies have identified distinctions between the immunological abnormalities associated with these diseases. Compared with individuals with Kawasaki disease, those with MIS-C have more severe lymphopenia, neutropenia, and thrombocytopenia, with increased expression of HLA-DR, a T cell activation marker, and CD57, a marker of CD4 ${ }^{+}$ $\mathrm{T}$ cell senescence ${ }^{118,119}$. Circulating levels of proteins associated with endothelial damage are higher in people with Kawasaki disease than in children with MIS-C ${ }^{119}$, reflecting the more frequent coronary artery involvement in Kawasaki disease ${ }^{118}$. These differences might explain why the great majority of coronary artery aneurysms in MIS-C resolve within 30 days, which contrasts with the persistent aneurysms associated with Kawasaki disease.

T cells. The function of T cells in MIS-C pathogenesis has also been investigated. One study found a strong enrichment for patrolling $\mathrm{CX} 3 \mathrm{CR} 1^{+} \mathrm{CD}^{+} \mathrm{T}$ cells, potentially contributing to the vascular manifestations of MIS- $\mathrm{C}^{90}$. $\mathrm{PD} 1^{+} \mathrm{CD} 39^{+} \mathrm{CD} 8^{+} \mathrm{T}$ cells were also increased in number, nominally higher than levels in adults with severe disease, indicative of prolonged activation and TCR signaling. Three separate studies, each with unique cohorts of children with MIS-C, identified pronounced skewing of the TCR $V_{\beta}$ repertoire ${ }^{91,125,126}$, with up to $24 \%$ of the sequenced clones expressing the TRBV11-2 V-region (V $\beta 21.3$ ) in one study ${ }^{126}$. The polyclonal nature of this expansion, highlighted in two studies ${ }^{125,126}$, is suggestive of superantigen-driven activation. Concordant with these findings, a superantigen-like motif has been identified in the SARS-CoV-2 spike protein sequence ${ }^{127}$. TCR skewing has been similarly identified in adults with severe COVID-19, although the $\mathrm{V}_{\beta}$ signatures identified in adults with severe COVID-19 seem to differ, thus far, from those identified in children with MIS-C ${ }^{127,128}$. By contrast, TRBV11-2 enrichment was not detected in individuals with mild SARS-CoV-2, Kawasaki disease, or toxic shock syndrome, or in age-matched febrile children ${ }^{91,125,126}$. After resolution of the disease, the repertoire of $\mathrm{T}$ cells from children with MIS-C re-normalized in distribution ${ }^{91}$. In light of the skewed TCR repertoires evident in some who have MIS-C, studies have investigated post-infectious sources of antigens ${ }^{122,129}$. Given the frequency of gastrointestinal symptoms in children with MIS-C $C^{6,108-110,113}$, the gastrointestinal tract has been hypothesized to function as a reservoir for SARS-CoV-2 (refs. ${ }^{122,129,130}$ ), as the virus is known to infect enterocytes ${ }^{131}$. Compared with healthy children and those with COVID-19, a cohort of 19 children with MIS-C investigated by Yonker et al. shows evidence of increased gastrointestinal permeability and circulating levels of SARS-CoV-2 spike protein and the S1 subunit, the latter of which correlated with TRBV11-2 expression ${ }^{122}$. Additional studies with larger, multicenter MIS-C cohorts are needed to understand the generalizability of these findings.

Antibodies. The serological features of MIS-C reflect the post-infectious timing of this disease. Children with MIS-C have IgG antibodies to SARS-CoV-2 with levels and neutralizing capacity similar to those of convalescent adults, irrespective of concomitant RT-PCR positivity ${ }^{21,120}$. Additionally, higher levels of total and neutralizing antibodies against the spike and RBD proteins have been found in children with MIS-C compared with those with acute COVID-19 (ref. ${ }^{132}$ ). These findings suggest that the nasopharyngeal load of virus reflects an antecedent, rather than acute, infection ${ }^{120,124}$. In addition to robust antibody responses to SARS-CoV-2, children with MIS-C have a concomitant expansion of antibodies against other common viruses, including common coronaviruses, influenza virus, RSV, and Epstein-Barr virus ${ }^{21}$. This diversity of antibodies is similar to the antibody profiles of non-hospitalized adults with mild to moderate COVID-19; by contrast, adults with severe COVID-19 requiring hospitalization have more limited expansion of antibodies to pathogens other than SARS-CoV-2 (refs. ${ }^{21,104}$ ). The cause of differences in the breadth of the serological response is not known, but investigators have hypothesized that attenuated immunity with aging leads to a narrower humoral response during SARS-CoV-2 infection ${ }^{104}$. Children with MIS-C have increased frequencies of proliferating $\mathrm{CD} 27^{+} \mathrm{CD} 38^{+}$plasmablasts, which correlates with increased percentages of proliferating $\mathrm{CD} 4^{+} \mathrm{T}$ cells-a feature that diminishes with age $\mathrm{e}^{90,91}$.

Differences in age-associated morbidity and mortality to subtypes of respiratory viruses have prompted investigations of how exposure to other coronavirus infections affects susceptibility to MIS-C. A small study of three children with MIS-C identified a lack of antibodies against the common human coronaviruses HKU1 and betacoronavirus 1 (ref. ${ }^{119}$ ). However, subsequent studies have identified comparable or increased levels of antibodies to HKU1 and other seasonal coronaviruses in children hospitalized with MIS-C, compared with those with COVID-19 and healthy children, with no consistent correlation across studies in the relationship between 
antibodies against seasonal coronaviruses and neutralizing antibodies to SARS-CoV-2 (refs. ${ }^{133,134}$ ).

The end-organ inflammation characteristic of MIS-C has prompted studies investigating the contributions of autoantibodies to the pathogenesis of MIS-C. Increased levels of circulating autoantibodies have been identified in children with MIS-C ${ }^{91,119,120}$, including in samples taken prior to administration of intravenous immunoglobulins. These autoantibodies recognize antigens in endothelial cells and the gastrointestinal tract, those associated with rheumatological disorders (systemic lupus erythematosus, Sjögren's disease, and idiopathic inflammatory myopathies), and those against widely expressed tissue antigens (MAPK2 and members of the casein kinase family) $)^{91,119,120}$. Additionally, two studies have found evidence of B cell clonal expansion in children with MIS- $C^{91,135}$, with the study by Porritt et al. showing that a higher frequency of immunoglobulin heavy-chain genes is associated with autoimmune disorders in individuals with TRBV11-2 expression ${ }^{135}$. Notably, studies of children with MIS-C have not identified autoantibodies against IFN or IFN-stimulated genes ${ }^{123,136}$, thus distinguishing autoantibodies in children with MIS-C from those detected in adults with severe COVID-19 (refs. ${ }^{64,78}$ ).

Genetic risk factors. Genetic risk factors associated with susceptibility to MIS-C are also distinct from those associated with severe COVID-19 in adults. Multiple studies of adults with severe COVID-19 have identified an association between severe COVID19 and loss-of-function variants of TLR7, a sensor for SARS-CoV-2 single-stranded RNA ${ }^{137-140}$. TLR7 signaling promotes IFN signaling, the secretion of IL- 6 , IL-1 $\beta$. and IL-23, and generation of the $\mathrm{T}_{\mathrm{H}} 17$ subset of helper $\mathrm{T}$ cells in response to viral infections ${ }^{141,142}$. Thus, reduced function of the protein encoded by TLR7, which is located on the $\mathrm{X}$ chromosome, is one mechanism contributing to the increased prevalence of severe COVID-19 in men compared with women ${ }^{143-145}$. In contrast to these risk variants for COVID-19, variants impairing negative regulators of inflammation have been detected in children with MIS-C ${ }^{146,147}$. These include SOCS1 (encoding suppressor of cytokine signaling 1), XIAP (encoding X-linked inhibitor of apoptosis), and CYBB (encoding cytochrome b-245, beta subunit) ${ }^{147}$. Even 7 months after recovery, transcriptional evidence of increased IFN and inflammatory signaling can be detected in individuals with these genetic risk variants, thus constituting a counterpoint to the reduced IFN signature associated with severe COVID-19 in adults. Extending beyond monogenic risk factors, studies have identified HLA class I alleles as factors potentially increasing a child's risk of MIS-C. In the study by Porritt et al., which had a predominance of Hispanic patients from the USA, four of seven patients with MIS-C shared the HLA class I alleles A02, B35, and $\mathrm{C} 04125^{125}$. By contrast, this enrichment for specific HLA class I alleles was not found in the sub-cohort of HLA-genotyped patients presented by Moreews et al., which was composed predominantly of children from Afro-Caribbean backgrounds ${ }^{126}$. Thus, larger cohort studies are necessary to quantify the association between HLA genotype on the susceptibility to MIS-C.

\section{Conclusion}

Many limitations of the studies presented here arise from challenges inherent to a pandemic. Children have smaller blood volumes than adults, which limits sample volumes, and the lower prevalence of SARS-CoV-2-associated complications in pediatric populations than in adult cohorts reduced study sample sizes ${ }^{148}$. The relative rarity of intubation required for children with COVID-19 prompted the use of nasopharyngeal samples as surrogates. The viral load, immune cell composition, and gene expression profiles of nasal samples and airway samples are distinct and not interchangeable ${ }^{69}$. The immunomodulatory treatments given to patients with MIS-C affects the transcriptional signature of immune cells, as seen in longitudinal studies of MIS- $C^{90,117,119}$. Historically, these types of limitations have been overcome by the use of animal models of disease to confirm the findings from patient studies. However, owing to interspecies differences in the susceptibility and response to SARS-CoV-2 infection, there are no animal models that recapitulate the two most severe manifestations of SARS-CoV-2 infection in humans: acute respiratory distress syndrome or MIS-C ${ }^{149}$.

Nevertheless, collectively, these studies show that the immune response of children and adults to mild SARS-CoV-2 infection are similar, but diverge after the development of severe disease (Fig. 3). Adults with severe COVID-19 might have loss of immune synchrony: $\mathrm{T}$ cell lymphopenia, reduced circulating follicular helper $\mathrm{T}$ cells, and impaired IFN-mediated signaling juxtaposed with high frequencies of activated SARS-CoV-2-specific T cells, robust humoral responses, and myeloid cell activation. By contrast, children with MIS-C exhibit multilineage immune cell activation beyond that which occurs in pediatric or adult COVID-19, evidenced by greater IFN and inflammasome signaling, widespread $\mathrm{T}$ cell activation, and activation of a broad humoral response reactive to common viruses as well as SARS-CoV-2. The association of severe COVID-19 in children and adults with pre-existing medical conditions underscores the contributions of these comorbidities to disease severity. Although studies have started to identify genetic risk factors for MIS-C, the constellation of factors that lead to MIS-C are mostly undefined. The spread of viral variants among unvaccinated pediatric populations may also change the spectrum of disease in children. The solutions to these questions will emerge as investigators continue to develop multicenter studies with larger cohorts alongside new tools for studying SARS-CoV-2 pathogenesis.

Received: 1 October 2021; Accepted: 13 December 2021; Published online: 1 February 2022

\section{References}

1. Gudbjartsson, D. F. et al. Spread of SARS-CoV-2 in the Icelandic population. N. Engl. J. Med. 382, 2302-2315 (2020).

2. Pan, X. et al. Asymptomatic cases in a family cluster with SARS-CoV-2 infection. Lancet Infect. Dis. 20, 410-411 (2020).

3. Jiehao, C. et al. A case series of children with 2019 novel coronavirus infection: clinical and epidemiological features. Clin. Infect. Dis. Publ. Infect. Dis. Soc. Am. 71, 1547-1551 (2020).

4. Lu, X. et al. SARS-CoV-2 infection in children. N. Engl. J. Med. 382, 1663-1665 (2020).

5. Grasselli, G. et al. Baseline characteristics and outcomes of 1591 patients infected with SARS-CoV-2 admitted to ICUs of the Lombardy region, Italy. J. Am. Med. Assoc. 323, 1574-1581 (2020).

6. Götzinger, F. et al. COVID-19 in children and adolescents in Europe: a multinational, multicentre cohort study. Lancet Child Adolesc. Health 4, 653-661 (2020).

7. Saatci, D. et al. Association between race and COVID-19 outcomes among 2.6 million children in England. JAMA Pediatr. 175, 928-938 (2021).

8. Viner, R. M. et al. Susceptibility to SARS-CoV-2 infection among children and adolescents compared with adults: a systematic review and meta-analysis. JAMA Pediatr. 175, 143-156 (2021).

9. Tsankov, B. K. et al. Severe COVID-19 infection and pediatric comorbidities: a systematic review and meta-analysis. Int. J. Infect. Dis. 103 246-256 (2021).

10. Preston, L. E. et al. Characteristics and disease severity of US children and adolescents diagnosed with COVID-19. JAMA Netw. Open 4, e215298-e215298 (2021)

11. Dewi, R. et al. Mortality in children with positive SARS-CoV-2 polymerase chain reaction test: Lessons learned from a tertiary referral hospital in Indonesia. Int. J. Infect. Dis. 107, 78-85 (2021).

12. Velásquez, S. R. et al. The toll of COVID-19 on African children: descriptive analysis on COVID-19-related morbidity and mortality among the pediatric population in sub-Saharan Africa. Int. J. Infect. Dis. 110, 457-465 (2021).

13. Shekerdemian, L. S. et al. Characteristics and outcomes of children with coronavirus disease 2019 (COVID-19) infection admitted to US and Canadian pediatric intensive care units. JAMA Pediatr. 174, 868-873 (2020).

14. Kenmoe, S. et al. Systematic review and meta-analysis of the prevalence of common respiratory viruses in children $<2$ years with bronchiolitis in the pre-COVID-19 pandemic era. PLoS ONE 15, e0242302 (2020). 
15. Wang, X. et al. Global burden of respiratory infections associated with seasonal influenza in children under 5 years in 2018: a systematic review and modelling study. Lancet Glob. Health 8, e497-e510 (2020).

16. Jones, T. C. et al. Estimating infectiousness throughout SARS-CoV-2 infection course. Science 373, eabi5273 (2021).

17. Chung, E. et al. Comparison of symptoms and RNA levels in children and adults with SARS-CoV-2 infection in the community setting. JAMA Pediatr. 175, e212025 (2021)

18. Madera, S. et al. Nasopharyngeal SARS-CoV-2 viral loads in young children do not differ significantly from those in older children and adults. Sci. Rep. 11, 3044 (2021).

19. Jia, R. et al. Mild cytokine elevation, moderate $\mathrm{CD} 4^{+} \mathrm{T}$ Cell response and abundant antibody production in children with COVID-19. Virol. Sin. 35 , 734-743 (2020).

20. Cohen, C. A. et al. SARS-CoV-2 specific $\mathrm{T}$ cell responses are lower in children and increase with age and time after infection. Nat. Commun. 12, 4678 (2021).

21. Bartsch, Y. C. et al. Humoral signatures of protective and pathological SARS-CoV-2 infection in children. Nat. Med. 27, 454-462 (2021).

22. Garrido, C. et al. Asymptomatic or mild symptomatic SARS-CoV-2 infection elicits durable neutralizing antibody responses in children and adolescents. JCI Insight 6, e150909 (2021).

23. Yang, H. S. et al. Association of age with SARS-CoV-2 antibody response. JAMA Netw. Open 4, e214302-e214302 (2021).

24. Méndez-Echevarría, A. et al. Long-term persistence of anti-SARS-CoV-2 antibodies in a pediatric population. Pathogens 10, 700 (2021).

25. Oygar, P. D. et al. Longitudinal follow-up of antibody responses in pediatric patients with COVID-19 up to 9 months after infection. Pediatr. Infect. Dis. J. 40, e294-e299 (2021).

26. Delahoy, M. J. Hospitalizations associated with COVID-19 among children and adolescents - COVID-NET, 14 states, March 1, 2020-August 14, 2021. MMWR Morb. Mortal. Wkly. Rep. 70, 1255-1260 (2021).

27. Pierce, C. A. et al. Immune responses to SARS-CoV-2 infection in hospitalized pediatric and adult patients. Sci. Transl. Med. 12 eabd5487 (2020).

28. Laxminarayan, R. et al. Epidemiology and transmission dynamics of COVID-19 in two Indian states. Science 370, 691-697 (2020).

29. Centers for Disease Control and Prevention. Weekly Updates by Select Demographic and Geographic Characteristics - Provisional Death Counts for Coronavirus Disease 2019 (COVID-19). National Center for Health Statistics https://www.cdc.gov/nchs/nvss/vsrr/covid_weekly/index.htm (2021).

30. Karakoc Aydiner, E. et al. Adverse COVID-19 outcomes in immune deficiencies: Inequality exists between subclasses. Allergy 77, 282-295 (2021).

31. Delavari, S. et al. Impact of SARS-CoV-2 pandemic on patients with primary immunodeficiency. J. Clin. Immunol. 41, 345-355 (2021).

32. Esenboga, S. et al. COVID-19 in patients with primary immunodeficiency. J. Clin. Immunol. 41, 1515-1522 (2021).

33. Meyts, I. et al. Coronavirus disease 2019 in patients with inborn errors of immunity: an international study. J. Allergy Clin. Immunol. 147, 520-531 (2020).

34. Shaunak, M. et al. COVID-19 symptom surveillance in immunocompromised children and young people in the UK: a prospective observational cohort study. BMJ Open 11, e044899 (2021).

35. Zhou, F. et al. Clinical course and risk factors for mortality of adult inpatients with COVID-19 in Wuhan, China: a retrospective cohort study. Lancet 395, 1054-1062 (2020).

36. $\mathrm{Li}, \mathrm{X}$. et al. Risk factors for severity and mortality in adult COVID-19 inpatients in Wuhan. J. Allergy Clin. Immunol. 146, 110-118 (2020).

37. Williamson, E. J. et al. Factors associated with COVID-19-related death using OpenSAFELY. Nature 584, 430-436 (2020).

38. Noz, M. P. et al. Trained immunity characteristics are associated with progressive cerebral small vessel disease. Stroke 49, 2910-2917 (2018).

39. Netea, M. G. et al. Defining trained immunity and its role in health and disease. Nat. Rev. Immunol. 20, 375-388 (2020)

40. Berbudi, A., Rahmadika, N., Tjahjadi, A. I. \& Ruslami, R. Type 2 diabetes and its impact on the immune system. Curr. Diabetes Rev. 16 442-449 (2020).

41. Sandoval, M., Nguyen, D. T., Vahidy, F. S. \& Graviss, E. A. Risk factors for severity of COVID-19 in hospital patients age 18-29 years. PLoS ONE 16, e0255544 (2021)

42. Wan, Y., Shang, J., Graham, R., Baric, R. S. \& Li, F. Receptor recognition by the novel coronavirus from wuhan: an analysis based on decade-long structural studies of SARS coronavirus. J. Virol. 94, e00127-20 (2020).

43. Zhou, P. et al. A pneumonia outbreak associated with a new coronavirus of probable bat origin. Nature 579, 270-273 (2020).

44. Shang, J. et al. Structural basis of receptor recognition by SARS-CoV-2. Nature 581, 221-224 (2020).
45. Walls, A. C. et al. Structure, function, and antigenicity of the SARS-CoV-2 spike glycoprotein. Cell 181, 281-292.e6 (2020).

46. Ou, X. et al. Characterization of spike glycoprotein of SARS-CoV-2 on virus entry and its immune cross-reactivity with SARS-CoV. Nat. Commun. 11, 1620 (2020).

47. Schuler, B. A. et al. Age-determined expression of priming protease TMPRSS2 and localization of SARS-CoV-2 in lung epithelium. J. Clin. Invest. 131, e140766 (2021).

48. Wang, A. et al. Single-cell multiomic profiling of human lungs reveals cell-type-specific and age-dynamic control of SARS-CoV2 host genes. eLife 9, e62522 (2020)

49. Letko, M., Marzi, A. \& Munster, V. Functional assessment of cell entry and receptor usage for SARS-CoV-2 and other lineage B betacoronaviruses. Nat. Microbiol. 5, 562-569 (2020).

50. Hoffmann, M. et al. SARS-CoV-2 cell entry depends on ACE2 and TMPRSS2 and is blocked by a clinically proven protease inhibitor. Cell 181, 271-280 (2020).

51. Muus, C. et al. Single-cell meta-analysis of SARS-CoV-2 entry genes across tissues and demographics. Nat. Med. 27, 546-559 (2021).

52. Pierce, C. A. et al. Natural mucosal barriers and COVID-19 in children. JCI Insight 6, e148694 (2021).

53. Bunyavanich, S., Do, A. \& Vicencio, A. Nasal gene expression of angiotensin-converting enzyme 2 in children and adults. J. Am. Med. Assoc. 323, 2427-2429 (2020)

54. Heald-Sargent, T. et al. Age-related differences in nasopharyngeal severe acute respiratory syndrome coronavirus 2 (SARS-CoV-2) levels in patients with mild to moderate coronavirus disease 2019 (COVID-19). JAMA Pediatr. 174, 902 (2020).

55. Fajnzylber, J. et al. SARS-CoV-2 viral load is associated with increased disease severity and mortality. Nat. Commun. 11, 5493 (2020).

56. Argyropoulos, K. V. et al. Association of initial viral load in severe acute respiratory syndrome coronavirus 2 (SARS-CoV-2) patients with outcome and symptoms. Am. J. Pathol. 190, 1881-1887 (2020).

57. Yang, Q. et al. Just $2 \%$ of SARS-CoV-2-positive individuals carry $90 \%$ of the virus circulating in communities. Proc. Natl Acad. Sci. USA 118, e2104547118 (2021)

58. Aykac, K. et al. The association of viral load and disease severity in children with COVID-19. J. Med. Virol. 93, 3077-3083 (2021).

59. Kim, C. et al. COVID-19 infection with asymptomatic or mild disease severity in young patients: clinical course and association between prevalence of pneumonia and viral load. PLOS ONE 16, e0250358 (2021)

60. Zheng, M. et al. TLR2 senses the SARS-CoV-2 envelope protein to produce inflammatory cytokines. Nat. Immunol. 22, 829-838 (2021).

61. Zhao, Y. et al. SARS-CoV-2 spike protein interacts with and activates TLR4. Cell Res. 31, 818-820 (2021).

62. Anaeigoudari, A., Mollaei, H. R., Arababadi, M. K. \& Nosratabadi, R. Severe acute respiratory syndrome coronavirus 2 : the role of the main components of the innate immune system. Inflammation 44, 2151-2169 (2021).

63. Xia, H. \& Shi, P.-Y. Antagonism of type I interferon by severe acute respiratory syndrome coronavirus 2. J. Interferon Cytokine Res. 40, 543-548 (2020).

64. Bastard, P. et al. Autoantibodies against type I IFNs in patients with life-threatening COVID-19. Science 370, eabd4585 (2020).

65. Lemarquis, A. et al. Severe COVID-19 in an APS1 patient with interferon autoantibodies treated with plasmapheresis. J. Allergy Clin. Immunol. 148, 96-98 (2021).

66. Bastard, P. et al. Preexisting autoantibodies to type I IFNs underlie critical COVID-19 pneumonia in patients with APS-1. J. Exp. Med. 218, e20210554 (2021).

67. Hadjadj, J. et al. Impaired type I interferon activity and inflammatory responses in severe COVID-19 patients. Science 369, 718-724 (2020)

68. Arunachalam, P. S. et al. Systems biological assessment of immunity to mild versus severe COVID-19 infection in humans. Science 369, 1210-1220 (2020)

69. Broggi, A. et al. Type III interferons disrupt the lung epithelial barrier upon viral recognition. Science 369, 706-712 (2020).

70. Weisberg, S. P. et al. Distinct antibody responses to SARS-CoV-2 in children and adults across the COVID-19 clinical spectrum. Nat. Immunol. 22, 25-31 (2021)

71. Nguyen, M. et al. Acquisition of adult-like TLR4 and TLR9 responses during the first year of life. PLoS One 5, e10407 (2010).

72. Holt, P. G. et al. Developmental regulation of type 1 and type 3 interferon production and risk for infant infections and asthma development. $J$. Allergy Clin. Immunol. 143, 1176-1182.e5 (2019).

73. Molony, R. D. et al. Aging impairs both primary and secondary RIG-I signaling for interferon induction in human monocytes. Sci. Signal. 10, eaan2392 (2017) 
74. Li, G., Ju, J., Weyand, C. M. \& Goronzy, J. J. Age-associated failure to adjust type I IFN receptor signaling thresholds after T cell activation. J. Immunol. 195, 865-874 (2015).

75. Le Page, A. et al. Downregulation of inhibitory SRC homology 2 domain-containing phosphatase-1 (SHP-1) leads to recovery of T cell responses in elderly. Cell Commun. Signal. 12, 2 (2014).

76. Macal, M. et al. Self-renewal and toll-like receptor signaling sustain exhausted plasmacytoid dendritic cells during chronic viral infection. Immunity 48, 730-744.e5 (2018).

77. Zuniga, E. I., Liou, L.-Y., Mack, L., Mendoza, M. \& Oldstone, M. B. A Persistent virus infection inhibits type I interferon production by plasmacytoid dendritic cells to facilitate opportunistic infections. Cell Host Microbe 4, 374-386 (2008).

78. Wijst, M. G. Pvander et al. Type I interferon autoantibodies are associated with systemic immune alterations in patients with COVID-19. Sci. Transl. Med. 13, eabh2624 (2021).

79. Chen, Z. \& John Wherry, E. T cell responses in patients with COVID-19. Nat. Rev. Immunol. 20, 529-536 (2020).

80. Bains, I., Thiébaut, R., Yates, A. J. \& Callard, R. Quantifying thymic export: combining models of naive $\mathrm{T}$ cell proliferation and TCR excision circle dynamics gives an explicit measure of thymic output. J. Immunol. 183, 4329-4336 (2009).

81. Kumar, B. V., Connors, T. J. \& Farber, D. L. Human T cell development, localization, and function throughout life. Immunity 48, 202-213 (2018)

82. Mateus, J. et al. Selective and cross-reactive SARS-CoV-2 T cell epitopes in unexposed humans. Science 370, 89-94 (2020).

83. Elyahu, Y. et al. Aging promotes reorganization of the CD4 $\mathrm{T}$ cell landscape toward extreme regulatory and effector phenotypes. Sci. Adv. 5, eaaw8330 (2019)

84. Tu, W. \& Rao, S. Mechanisms underlying T cell immunosenescence: aging and cytomegalovirus infection. Front. Microbiol. 7, 2111 (2016).

85. Nikolich-Žugich, J. Ageing and life-long maintenance of T-cell subsets in the face of latent persistent infections. Nat. Rev. Immunol. 8, 512-522 (2008).

86. Turner, J. S. et al. SARS-CoV-2 infection induces long-lived bone marrow plasma cells in humans. Nature 595, 421-425 (2021).

87. Mathew, D. et al. Deep immune profiling of COVID-19 patients reveals distinct immunotypes with therapeutic implications. Science 369, eabc8511 (2020)

88. Mudd, P. A. et al. Distinct inflammatory profiles distinguish COVID-19 from influenza with limited contributions from cytokine storm. Sci. Adv. 6, eabe3024 (2020).

89. Kuri-Cervantes, L. et al. Comprehensive mapping of immune perturbations associated with severe COVID-19. Sci. Immunol. 5, eabd7114 (2020).

90. Vella, L. A. et al. Deep immune profiling of MIS-C demonstrates marked but transient immune activation compared to adult and pediatric COVID-19. Sci. Immunol. 6, eabf7570 (2021).

91. Ramaswamy, A. et al. Immune dysregulation and autoreactivity correlate with disease severity in SARS-CoV-2-associated multisystem inflammatory syndrome in children. Immunity 54, 1083-1095.e7 (2021).

92. Pollard, A. J., Perrett, K. P. \& Beverley, P. C. Maintaining protection against invasive bacteria with protein-polysaccharide conjugate vaccines. Nat. Rev. Immunol. 9, 213-220 (2009).

93. Nielsen, S. C. A. et al. Shaping of infant B cell receptor repertoires by environmental factors and infectious disease. Sci. Transl. Med. 11, eaat2004 (2019).

94. Wendel, B. S. et al. Accurate immune repertoire sequencing reveals malaria infection driven antibody lineage diversification in young children. Nat. Commun. 8, 531 (2017).

95. Olin, A. et al. Stereotypic immune system development in newborn children. Cell 174, 1277-1292.e14 (2018)

96. Khan, T. et al. Distinct antibody repertoires against endemic human coronaviruses in children and adults. JCI Insight 6, e144499 (2021).

97. Goodwin, E. et al. Infants infected with respiratory syncytial virus generate potent neutralizing antibodies that lack somatic hypermutation. Immunity 48, 339-349.e5 (2018).

98. Gilman, M. S. A. et al. Rapid profiling of RSV antibody repertoires from the memory B cells of naturally infected adult donors. Sci. Immunol. 1, eaaj1879-eaaj1879 (2016)

99. Meade, P. et al. Influenza virus infection induces a narrow antibody response in children but a broad recall response in adults. mBio 11 , e03243-19 (2020)

100. Ranjeva, S. et al. Age-specific differences in the dynamics of protective immunity to influenza. Nat. Commun. 10, 1660 (2019).

101. Gostic, K. M., Ambrose, M., Worobey, M. \& Lloyd-Smith, J. O. Potent protection against $\mathrm{H} 5 \mathrm{~N} 1$ and $\mathrm{H} 7 \mathrm{~N} 9$ influenza via childhood hemagglutinin imprinting. 354, 722-726 (2016).

102. Arevalo, C. P. et al. Original antigenic sin priming of influenza virus hemagglutinin stalk antibodies. Proc. Natl Acad. Sci. USA 117, 17221-17227 (2020).
103. Aydillo, T. et al. Immunological imprinting of the antibody response in COVID-19 patients. Nat. Commun. 12, 3781 (2021).

104. Shrock, E. et al. Viral epitope profiling of COVID-19 patients reveals cross-reactivity and correlates of severity. Science 370, eabd4250 (2020).

105. Sasson, J. M. et al. Diverse humoral immune responses in younger and older adult COVID-19 patients. mBio 12, e01229-21 (2021).

106. Verdoni, L. et al. An outbreak of severe Kawasaki-like disease at the Italian epicentre of the SARS-CoV-2 epidemic: an observational cohort study. Lancet 395, 1771-1778 (2020).

107. Riphagen, S., Gomez, X., Gonzalez-Martinez, C., Wilkinson, N. \& Theocharis, P. Hyperinflammatory shock in children during COVID-19 pandemic. Lancet 395, 1607-1608 (2020).

108. Viner, R. M. \& Whittaker, E. Kawasaki-like disease: emerging complication during the COVID-19 pandemic. Lancet 395, 1741-1743 (2020).

109. Whittaker, E. et al. Clinical characteristics of 58 children with a pediatric inflammatory multisystem syndrome temporally associated with SARS-CoV-2. J. Am. Med. Assoc. 324, 259-269 (2020).

110. Feldstein, L. R. et al. Multisystem inflammatory syndrome in US children and adolescents. N. Engl. J. Med. 383, 334-346 (2020).

111. Dufort, E. M. et al. Multisystem inflammatory syndrome in children in New York State. N. Engl. J. Med. 383, 347-358 (2020).

112. Centers for Disease Control and Prevention. Emergency preparedness and response: multisystem inflammatory syndrome in children (MIS-C) associated with coronavirus disease 2019 (COVID-19). CDC Emergency Preparedness and Response https://emergency.cdc.gov/han/2020/han00432. asp (2021).

113. Feldstein, L. R. et al. Characteristics and outcomes of US children and adolescents with multisystem inflammatory syndrome in children (MIS-C) compared with severe acute COVID-19. J. Am. Med. Assoc. 325, 1074 (2021).

114. Abrams, J. Y. et al. Factors linked to severe outcomes in multisystem inflammatory syndrome in children (MIS-C) in the USA: a retrospective surveillance study. Lancet Child Adolesc. Health 5, 323-331 (2021).

115. Sancho-Shimizu, V. et al. SARS-CoV-2-related MIS-C: a key to the viral and genetic causes of Kawasaki disease? J. Exp. Med. 218, e20210446 (2021).

116. Kwak, J. H., Lee, S.-Y. \& Choi, J.-W. Clinical features, diagnosis, and outcomes of multisystem inflammatory syndrome in children associated with coronavirus disease 2019. Clin. Exp. Pediatr. 64, 68-75 (2020).

117. Carter, M. J. et al. Peripheral immunophenotypes in children with multisystem inflammatory syndrome associated with SARS-CoV-2 infection. Nat. Med. 26, 1701-1707 (2020)

118. Lee, P. Y. et al. Distinct clinical and immunological features of SARS-CoV2 -induced multisystem inflammatory syndrome in children. J. Clin. Invest. 130, 5942-5950 (2020)

119. Consiglio, C. R. et al. The immunology of multisystem inflammatory syndrome in children with COVID-19. Cell 183, 968-981.e7 (2020).

120. Gruber, C. N. et al. Mapping systemic inflammation and antibody responses in multisystem inflammatory syndrome in children (MIS-C). Cell 183, 982-995.e14 (2020).

121. Chang, J. C. et al. Skewed cytokine responses rather than the magnitude of the cytokine storm may drive cardiac dysfunction in multisystem inflammatory syndrome in children. J. Am. Heart Assoc. 10, e021428 (2021)

122. Yonker, L. M. et al. Multisystem inflammatory syndrome in children is driven by zonulin-dependent loss of gut mucosal barrier. J. Clin. Invest. 131, e149633 (2021).

123. de Cevins, C. et al. A monocyte/dendritic cell molecular signature of SARS-CoV-2-related multisystem inflammatory syndrome in children with severe myocarditis. Med. NY 2, 1072-1092 (2021).

124. Diorio, C. et al. Multisystem inflammatory syndrome in children and COVID-19 are distinct presentations of SARS-CoV-2. J. Clin. Invest. 130, 5967-5975 (2020)

125. Porritt, R. A. et al. HLA class I-associated expansion of TRBV11-2 T cell in multisystem inflammatory syndrome in children. J. Clin. Invest. 131, e146614 (2021).

126. Moreews, $\mathrm{M}$. et al. Polyclonal expansion of TCR Vb $21.3^{+} \mathrm{CD} 4^{+}$and $\mathrm{CD} 8^{+}$ $\mathrm{T}$ cells is a hallmark of multisystem inflammatory syndrome in children. Sci. Immunol. 6, eabh1516 (2021).

127. Cheng, M. H. et al. Superantigenic character of an insert unique to SARS-CoV-2 spike supported by skewed TCR repertoire in patients with hyperinflammation. Proc. Natl Acad. Sci. 117, 25254-25262 (2020).

128. Notarbartolo, S. et al. Integrated longitudinal immunophenotypic, transcriptional, and repertoire analyses delineate immune responses in patients with COVID-19. Sci. Immunol. 6, eabg5021 (2021).

129. Josyabhatla, R. et al. Recognizing a MIS-CHievous cause of acute viral gastroenteritis. Front. Pediatr. 9, 1123 (2021).

130. Noval Rivas, M., Porritt, R. A., Cheng, M. H., Bahar, I. \& Arditi, M. COVID-19-associated multisystem inflammatory syndrome in children (MIS-C): a novel disease that mimics toxic shock syndrome-the superantigen hypothesis. J. Allergy Clin. Immunol. 147, 57-59 (2021) 
131. Lamers, M. M. et al. SARS-CoV-2 productively infects human gut enterocytes. Science 369, 50-54 (2020).

132. Anderson, E. M. et al. Severe acute respiratory syndrome-coronavirus-2 (SARS-CoV-2) antibody responses in children with multisystem inflammatory syndrome in children (MIS-C) and mild and severe coronavirus disease 2019 (COVID-19). J. Pediatr. Infect. Dis. Soc. 10, 669-673 (2021).

133. Lapp, S. A. et al. Original antigenic sin responses to Betacoronavirus spike proteins are observed in a mouse model, but are not apparent in children following SARS-CoV-2 infection. PLoS ONE 16, e0256482 (2021).

134. Sermet-Gaudelus, I. et al. Prior infection by seasonal coronaviruses, as assessed by serology, does not prevent SARS-CoV-2 infection and disease in children, France, April to June 2020. Euro Surveill. 26, 2001782 (2021).

135. Porritt, R. A. et al. The autoimmune signature of hyperinflammatory multisystem inflammatory syndrome in children. J. Clin. Invest. 131, e151520 (2021).

136. Combes, A. J. et al. Global absence and targeting of protective immune states in severe COVID-19. Nature 591, 124-130 (2021).

137. van der Made, C. I. et al. Presence of genetic variants among young men with severe COVID-19. J. Am. Med. Assoc. 324, 663 (2020).

138. Asano, T. et al. X-linked recessive TLR7 deficiency in $\sim 1 \%$ of men under 60 years old with life-threatening COVID-19. Sci. Immunol. 6, eabl4348 (2021).

139. Fallerini, C. et al. Association of Toll-like receptor 7 variants with life-threatening COVID-19 disease in males: findings from a nested case-control study. eLife 10, e67569 (2021).

140. Solanich, X. et al. Genetic screening for TLR7 variants in young and previously healthy men with severe COVID-19. Front. Immunol. 12, 2965 (2021).

141. Swiecki, M. \& Colonna, M. The multifaceted biology of plasmacytoid dendritic cells. Nat. Rev. Immunol. 15, 471-485 (2015).

142. de Marcken, M., Dhaliwal, K., Danielsen, A. C., Gautron, A. S. \& Dominguez-Villar, M. TLR7 and TLR8 activate distinct pathways in monocytes during RNA virus infection. Sci. Signal. 12, eaaw1347 (2019).

143. Ioannou, G. N. et al. Risk factors for hospitalization, mechanical ventilation, or death among 10131 US veterans with SARS-CoV-2 infection. JAMA Netw. Open 3, e2022310-e2022310 (2020).

144. Peckham, H. et al. Male sex identified by global COVID-19 meta-analysis as a risk factor for death and ITU admission. Nat. Commun. 11, 6317 (2020)
145. Nguyen, N. T. et al. Male gender is a predictor of higher mortality in hospitalized adults with COVID-19. PLoS ONE16, e0254066 (2021).

146. Lee, P. Y. et al. Immune dysregulation and multisystem inflammatory syndrome in children (MIS-C) in individuals with haploinsufficiency of SOCS1. J. Allergy Clin. Immunol. 146, 1194-1200.e1 (2020).

147. Chou, J. et al. Mechanisms underlying genetic susceptibility to multisystem inflammatory syndrome in children (MIS-C). J. Allergy Clin. Immunol. 148 732-738.e1 (2021)

148. Bourgeois, F. T., Avillach, P. \& Turner, M. A. The urgent need for research coordination to advance knowledge on COVID-19 in children. Pediatr. Res. 90, 250-252 (2020).

149. Muñoz-Fontela, C. et al. Animal models for COVID-19. Nature 586, 509-515 (2020).

\section{Acknowledgements}

The authors are supported by the National Institute of Diabetes and Digestive and Kidney Diseases (R01DK130465 to J. C.) and the National Institute of Allergy and Infectious Diseases (U01AI144616 to P. T., R01AI154470 to A. G. R., and R01AI139633-04S1 to J. C.)

\section{Author contributions}

All authors wrote and revised the manuscript.

\section{Competing interests}

P. G. T. is on the scientific advisory board of Immunoscape and Cytoagents. The other authors declare no competing interests.

\section{Additional information}

Correspondence should be addressed to Adrienne G. Randolph.

Peer review information Nature Immunology thanks Moshe Arditi, Marc Tebruegge, and Laura Vella for their contribution to the peer review of this work. Nick Bernard was the primary editor on this article and managed its editorial process and peer review in collaboration with the rest of the editorial team.

Reprints and permissions information is available at www.nature.com/reprints.

Publisher's note Springer Nature remains neutral with regard to jurisdictional claims in published maps and institutional affiliations.

(C) Springer Nature America, Inc. 2022 